\title{
LA FINANCIACIÓN DEL TERRORISMO DE AL QAIDA: MITOS Y REALIDADES
}

\author{
NIKOS PASSAS ${ }^{1}$ \\ ANDREA GimÉnEZ-SALINAS Framis ${ }^{2}$
}

Resumen: Este artículo ofrece, en primer lugar, una descripción de las fuentes de financiación del terrorismo de los grupos relacionados e inspirados en al Qaida. En dicha descripción, se contrasta la información procedente de la literatura con los datos empíricos revisados en una investigación llevada a cabo por los autores. A continuación, se describen los modos de transferencia de fondos más utilizados por los grupos yihadistas, con especial referencia al hawala, su funcionamiento y sus dificultades de control. Por último y a la luz de la financiación de algunos atentados realizados en nuestro territorio y en el exterior, se ofrecen algunas pautas o recomendaciones sobre el control de la financiación del terrorismo, teniendo en cuenta su especificidad respecto a otros fenómenos semejantes ${ }^{3}$.

Palabras clave: financiación del terrorismo, terrorismo.

1 Profesor Northeastern University.

2 Profesora de la Universidad Autónoma de Madrid y colaboradora de la investigación «Terrorist finance and the nexus with Transnational Organized Crime: Commodity Trade and the Social Organization of al-Qaida Groups», financiada por el Departamento de Justicia de EEUU.

3 Queremos agradecer especialmente la ayuda de Rosemary Barberet y de Ignacio Gordillo en esta investigación, ya que sin su apoyo y colaboración no hubiera sido posible. Sin embargo, nuestra deuda con ellos no les hace responsables del contenido de este artículo que a los autores unicamente les corresponde. 


\section{La financiación del terrorismo: una prioridad en la lucha contra el terror}

La financiación del terrorismo se ha convertido, especialmente a partir de los atentados del 11 de septiembre, en una prioridad en las agendas políticas nacionales de algunos Estados y en una prioridad internacional de carácter urgente. Sin embargo y a pesar de la imagen que se ofrece a través de los medios de comunicación y de las autoridades gubernamentales, la lucha contra la financiación del terrorismo no está dando los resultados esperados. En un inicio, la premisa de la que se partía era muy sencilla: si seguimos el rastro del dinero, podremos cortar los medios a través de los cuales se alimentan y desarrollan los grupos terroristas, y la consecuencia lógica será la progresiva decadencia y debilidad de los mismos para conseguir finalmente su inactividad. Medidas urgentes se han puesto en marcha desde los atentados del $11 \mathrm{~S}$ para mejorar el control financiero y económico relacionado con el terrorismo. Después de un tiempo, los resultados no han sido tan optimistas.

Evidentemente, el frente económico es un frente de acción que debe tenerse en cuenta a la hora de frenar la actividad antiterrorista pero no el único. Dicho frente es estéril si no va unido a otros frentes como son el apoyo religioso, social y cultural que solidariza y fortalece a estos grupos que utilizan el terror como herramienta de expresión. En tanto que frente económico de lucha, el rastreo del dinero y el control de las redes de apoyo financiero y logístico es una herramienta útil para la lucha antiterrorista por diferentes motivos. El bloqueo de la financiación conduce a una disminución del impacto y gravedad de los actos terroristas (Passas, 2003a), priva a los terroristas de medios para viajar, comunicarse, procurarse equipamiento y perpetrar atentados, obliga a cambiar las estrategias y modus operandi de los terroristas y así incrementa las oportunidades de detección de militantes y, por último, fomenta la mejora de la inteligencia aplicada al sector financiero, lo que puede beneficiar enormemente a otros problemas como la lucha contra el crimen organizado o el blanqueo de capitales.

Sin embargo, las medidas que se han adoptado ${ }^{4}$ han sido más el resultado de una política de urgencia y de la necesidad de establecer

${ }^{4}$ Convención de Naciones Unidas de 1999 para la supresión de la financiación del terrorismo, las Recomendaciones especiales del FATF, del Banco mundial y las prácticas de evaluación del IMF así como las leyes y normas cuya finalidad con la identificación, bloqueo y confiscación de fondos presuntamente relacionados con el apoyo a actividades terroristas. 
medidas de choque a toda costa, que el producto de un análisis riguroso de la realidad y del fenómeno al que nos estamos enfrentando. Todavía quedan importantes pasos por dar hacia la mejora del conocimiento del problema, sus dimensiones, su forma y capacidad de movimientos, sus posibilidades de actuación. Es necesario incrementar los esfuerzos para identificar mejor los modos de financiación, modus operandi, estrategias alternativas, para así adecuar mejor las políticas de control y prevención, evitando daños colaterales y abusos contra terceros inocentes (Passas, 2003). Al no haber identificado y definido el objetivo al que estamos dirigiendo nuestro control, las políticas que se han desarrollado son, en muchos casos, exageradas y peligrosamente preventivas provocando errores y excesivos daños colaterales.

El objetivo de este artículo es conocer un poco más sobre la financiación del terrorismo relacionado con al Qaida para así identificar las políticas de control más idóneas y ajustadas al fenómeno. Para ello, dividiremos nuestra exposición en dos partes. La primera es puramente descriptiva y pretende ahondar sobre lo que conocemos desde la literatura sobre la financiación del terrorismo de al Qaida, sus fuentes de financiación, los modos de transferencia de fondos utilizados con mayor frecuencia por estos grupos. La segunda parte del artículo responde a la voluntad de apuntar ciertos elementos al debate sobre el control de la financiación del terrorismo, partiendo del conocimiento empírico del fenómeno que tenemos hasta el momento ${ }^{5}$.

\section{Fuentes de financiación del terrorismo de al Qaida}

El origen de la financiación del terrorismo ha cambiado profundamente a partir del final de la Guerra fría, cuando se inició una progresiva reducción de la financiación "pública» de las organizaciones terroristas. Durante la guerra fría se dieron abundantes casos en los que los gobiernos aportaron apoyo tanto logístico como financiero a grupos terroristas. Además de casos conocidos como los ocurridos en Francia, EEUU o la URSS, otros países más pequeños también han contribuido en alguna que otra etapa a financiar y dar apoyo a grupos terroristas (Afganistán, Cuba, Irán, Irak, Libia, Corea del Norte, Pakistán, Arabia Saudita, Turquía).

${ }^{5}$ La información contenida en este artículo se basa en los resultados de la mencionada investigación: «Terrorist finance and the nexus with Transnational Organized Crime: Commodity Trade and the Social Organization of al-Qaida Groups» dirigida por el Prof. Nikos Passas. 
En las últimas décadas, la fuente pública o gubernamental de financiación de los grupos terroristas ha disminuido considerablemente aunque todavía tenemos ejemplos de organizaciones como Hamas, Hezbollah, Hizbul Mujahideen, IMU, Yihad islámica, etc. que se benefician todavía de este amparo económico estatal. Sin embargo, esta regresión en la esponsorización de organizaciones terroristas por parte de los Estados no ha conducido a una disminución del número de grupos radicales o terroristas activos, o a una merma en sus capacidades de actuación. El apoyo estatal ha sido progresivamente sustituido por fuentes alternativas de financiación como las fuentes legales, la delincuencia organizada o la delincuencia común ${ }^{6}$.

Diferentes fuentes de financiación han sido identificadas en la literatura como el origen de los fondos que sustentan las actividades de grupos terroristas relacionados con al Qaida. Estas fuentes pueden ser legales o ilegales y, es precisamente cuando se procede a esta diferenciación, cuando nos encontramos las diferencias conceptuales entre financiación del terrorismo y otras formas irregulares de utilización del sistema financiero. Es, en efecto, la posibilidad de que el origen de los fondos utilizados por los terroristas sea legal, lo que diferencia el concepto de financiación del terrorismo de otros conceptos como el blanqueo de capitales. El blanqueo de capitales es un proceso a través del cual el origen ilegal de los fondos se disfraza inyectando, a través de distintos mecanismos, esos mismos fondos en el sistema financiero legal. Sin embargo, en la financiación del terrorismo, el origen de los fondos no necesariamente tiene que ser ilegal ni tampoco se requiere obligatoriamente un proceso de blanqueo de dinero cuando los fondos van a ser destinados a fines ilegales o terroristas ${ }^{7}$. En estos casos, la financiación del terrorismo funcionaría como el blanqueo de capitales pero a la inversa: un dinero de procedencia legal es utilizado para fines ilegales. Cuando los fondos son de procedencia ilegal, no es necesario ningún proceso de blanqueo ya que, como son destinados a financiar acciones ilegales, no necesitan pasar un proceso de legalización de los mismos. En este último caso, lo fundamental es ocultar el rastro del dinero.

${ }^{6}$ El crimen local es uno de las opciones de financiación más utilizadas por al Qaida en estos momentos, según un informe del UN Monitoring Team (2005 para 65, 94fff).

7 Esta es la razón por la que en España tenemos dos leyes distintas: La ley 19/1993, de 28 de diciembre sobre determinadas medidas de prevención del blanqueo de capitales y la Ley 12/2003 de prevención y bloqueo de la financiación del terrorismo. La primera de ellas no contemplaba en la definición de blanqueo de capitales la posibilidad de que los fondos tuvieran una procedencia legal y se pudieran destinar a actuaciones ilícitas, especialmente el terrorismo. 
A continuación describimos y comentamos las diferentes fuentes de financiación del terrorismo que se identifican en la literatura.

\section{Fondos procedentes de la beneficencia}

Este ha sido el aspecto más mediático de la financiación del terrorismo islámico y sobre el que se ha incidido más a nivel internacional. Importantes avances se están llevando a cabo para controlar el desvío de fondos procedentes de ONGs y organizaciones benéficas cuyos objetivos sociales y legítimos suelen ser la ayuda a la construcción de escuelas, mezquitas, centros culturales, la traducción de textos y demás servicios a la comunidad islámica. La mayoría de estas organizaciones están bien establecidas en las comunidades islámicas locales y están muy implicadas en la satisfacción legítima de las necesidades de estas comunidades musulmanas en sus países y en el extranjero. Sin embargo, en otros casos podrían ser una fuente de financiación por parte de la red de al Qaida para apoyar instituciones fundamentalistas radicales, escuelas u organizaciones sociales que ofrecen apoyo logístico, formación, reclutamiento y refugio a sus militantes ${ }^{8}$. Sin embargo, tenemos que ser muy cautos con esta fuente de financiación ya que, después de cinco años de investigación sobre ONGs presuntamente implicadas en la financiación de grupos y actos terroristas, ninguna evidencia se ha encontrado de las sospechas iniciales (Passas, 2006). En EEUU se han congelado fondos e investigado distintas ONGs hasta el día de hoy: Global Relief Foundation, Holy Land Foundation for Relief and Development, Benevolence International Foundation e Islamic American Relief Agency. Estas investigaciones no han dado los resultados esperados y no se ha podido probar ninguna vinculación de estas organizaciones con la financiación del terrorismo. En algunos casos, las medidas adoptadas para congelar los fondos han obligado a algunas organizaciones a terminar sus actividades ${ }^{9}$. Se procedió agresivamente contra las organizaciones benéficas islámicas y no se han podido demostrar vínculos estrechos con el terrorismo, con lo cual el daño que se ha hecho supera con creces lo que se pretendía evitar. Esto no quiere decir que este sector no pueda ser vulnerable, que no haya que estar vigilante respecto a sus actividades y la posible confusión de actividades legítimas e ilegítimas, no obstante, las medidas de choque que se apliquen deberían venir acompañadas de un mejor conocimiento de la realidad para evitar

\footnotetext{
8 Report of the Independent Task Force on Terrorism Financing, p. 12.

9 http: //www.ombwatch.org/pdfs/muslim_charities.pdf.
} 
daños innecesarios. Estas medidas tan agresivas lo único que sí consiguen es alimentar el resentimiento de las comunidades afectadas.

Además de las donaciones gestionadas a través de ONGs u organizaciones encargadas de realizar actividades de beneficencia existe otra fuente de financiación de este tipo de terrorismo que tiene su origen en contribuciones o donaciones privadas derivadas de la obligación de la comunidad musulmana a dedicar una parte de sus ingresos a la beneficencia, lo que se denomina Zakat. El Zakat normalmente representa un 2,5 por ciento de los ingresos de cada musulmán y el donante debe tener conocimiento del destino de su dinero. Estas donaciones son utilizadas por los grupos terroristas para recaudar fondos a nivel local, entre los vecinos o comerciantes más cercanos. Además de las donaciones zakat, que son obligatorias, existen también las donaciones voluntarias como el infaq y shadaqah que se efectúan en ocasiones concretas ${ }^{10}$.

\section{Empresas tapadera o fantasma}

Este modo de financiación no sólo es propio del terrorismo, también es muy utilizado por las redes de delincuencia organizada, blanqueo de capitales, etc. y no sólo actúa como fuente de ingresos sino también como forma de enmascarar el origen de los fondos y para encubrir la finalidad ilegal de un dinero procedente de fuentes legales o ilegales. Este es el caso típico de las empresas tapadera o empresas que reciben dinero de al Qaida o de procedencia ilegal para capitalizar sus inicios pero cuyo fin principal es generar ingresos o mezclar capital blanqueado con ingresos de negocios lícitos ${ }^{11}$. En el caso de las grupos inspirados por al Qaida en Europa hemos encontrado, en numerosos $\operatorname{casos}^{12}$, que empresas pequeñas y locales sirven como depósito de fondos de origen legal o ilegal para así destinar el dinero a otros fines legales o ilegales. También nos encontramos con otro tipo de empresas, las empresas fantasmas o que se establecen con una suma de capital mínimo y generan escasos beneficios con el fin principal de comprar material o encubrir aspectos relacionados con acciones terroristas.

${ }^{10}$ http: //en.wikipedia.org/wiki/Encyclopaedia_of_Islam.

11 Financial Action Task Force on Money Laundering. Report on Money Laundering Typologies, 2002-2003, p. 3.

12 Operación dátil y célula relacionada con Ansar Al Islam, ambos casos enjuiciados en España. 


\section{Inversiones de al Qaida en la región}

Tanto al Qaida como otros grupos terroristas utilizan negocios legítimos locales para financiarse, ej. IRA, ETA ${ }^{13}$, FARC, Hezbollah, Abu Nidal, etc. Uno de los sectores más utilizados por estas redes para invertir es el sector inmobiliario ${ }^{14}$. Sin embargo, estas empresas no sólo generan dinero y pueden ser utilizadas para obtener financiación, en muchas ocasiones también producen más pérdidas que ganancias (Naylor, 2002 y 2006).

En este tipo de negocios es donde se produce la inversión del modus operandi respecto al blanqueo de capitales. En estos casos, se trata de negocios legales a través de los cuales una parte o todo el dinero que se genera, se dedica a una actividad ilegal. El origen del dinero es legal y el destino del dinero es ilegal. Este proceso inverso al blanqueo de capitales es lo que caracteriza a la financiación del terrorismo. En este tipo de operaciones no es necesario un proceso de blanqueo de dinero ya que sólo se blanquea aquel dinero de procedencia ilegal que se quiere reintroducir en el sistema financiero. En la financiación del terrorismo adquiere más importancia su ocultación y, sobre todo, la ocultación de su movimiento. Para conseguir este efecto, se utilizan diferentes métodos que se expondrán más adelante, destinados a dificultar la trazabilidad y seguimiento de los fondos.

\section{Fuentes ilegales}

El acudir a fuentes ilegales de financiación es también una constante en la práctica terrorista, sobre todo a partir de la reducción de la financiación estatal a las organizaciones terroristas. La delincuen-

13 En 1992, a partir de la detención de la cúpula de ETA en Bidart, se descubrió un plan de financiación para las organizaciones dependientes del MLNV. Este plan de financiación se llama Udaletxe y consistía en un entramado de empresas que tenia como objetivo desvincular la financiación de las organizaciones del entorno de ETA de su directa aportación. El objetivo era crear un medio más seguro de financiar las organizaciones que formaban el MLNV y tener fuentes de financiación propias para asegurarse ingresos permanentes. Este plan ha sido gestionado y coordinado por ETA y KAS a través de una comisión denominada "Comisión de proyectos de Udaletxe». Las fuentes de financiación principales eran: txoznas o bares de fiestas, herriko tabernas, la venta de material como camisetas, pegatinas, etc, cuotas de miembros, rifas y loterías y la utilización de subvenciones del gobierno vasco. Los beneficios obtenidos por estas empresas y según el documento incautado sobre Reunión de responsables del Proyecto Udaletxe estaban divididos en 50\% para Herri Batasua, 30\% para Gestoras pro Amnistía y $20 \%$ para Kas (Conclusiones provisionales sumario 18/98 - Juzgado Central n. 5. Audiencia Nacional).

14 Report of the Independent Task Force on Terrorism Financing, p. 11. 
cia ha sido un recurso muy utilizado por grupos terroristas: la extorsión, el secuestro, el chantaje, la exigencia de impuestos revolucionarios han sido mecanismos utilizados por grupos terroristas para autofinanciarse. Los grupos vinculados o inspirados en al Qaida suelen autofinanciarse a través la delincuencia común o fraude de tarjetas de crédito y parece que existe una gran flexibilidad a la hora de pasar de acciones para procurar financiación a acciones operativas (Aristegui, 2004). Diferentes tipos de estafas son utilizados en distintos niveles también por los grupos terroristas. El GIA y el Grupo Salafista para la predicación y el combate $(\mathrm{GSPC})^{15}$ han sido acusados de usar regularmente la falsificación de tarjetas de crédito y de documentación como modo de financiación. Asimismo, Al Gammat al Islamiya, al Qaida, Hezbollah, el IRA, los grupos chechenos han estado implicados en la falsificación y contrabando de bienes y moneda. También se cometen otro tipo de estafas por estos grupos terroristas, las estafas comerciales, la evasión de impuestos, el fraude a las aseguradoras y el robo de cajeros automáticos.

El tráfico de inmigrantes o la inmigración ilegal parece que ha sido también un recurso utilizado por grupos terroristas para procurar financiación a sus miembros. Parece ser que ha sido utilizado por grupos terroristas en Sri Lanka (Schmid, 2003: 3), también el GIA, KLA y Jemaah Islamiya han afirmado haber utilizado esta actividad ilegal. Asimismo, se ha puesto de manifiesto por los resultados de una encuesta a 38 países que en la cuarta parte de estos países existe relación entre terrorismo y el tráfico de inmigrantes (Dandurand y Chin, 2004). Sin embargo, nuevamente las evidencias son débiles y habría que intentar tener pruebas empíricas más consistentes para poder afirmar la relación de estas actividades con el terrorismo de al Qaida o sus simpatizantes.

Ocurre lo mismo con la relación entre el terrorismo y el tráfico de drogas. Existe una fuerte controversia sobre la relación que existe entre ambas redes pese a que los medios de comunicación den siempre por supuesta esta simbiosis. Casos como el atentado de Madrid ponen de manifiesto esta posible relación, pero también existen muchas razones para pensar que la relación entre terroristas y traficantes de droga sean de tipo ocasional y no permanente, ya que sus objetivos, finalidades y actitudes frente al Estado son absolutamente distintos y,

${ }^{15}$ En una investigación policial conjunta entre España, Suiza y Francia (operación Green) todavía abierta, se ha desmantelado un estructura en Europa que financiaba al GSPC. Al parecer, los fondos procedentes principalmente de robos en domicilios han permitido financiar, al menos dos atentados en Argelia y Mauritania (http: //www.mir.es/DGRIS/Notas_Prensa/Guardia_Civil/2006/np060904.htm). 
en muchos casos, una relación muy intensa pondría en peligro los objetivos y la supervivencia de las organizaciones terroristas. Las relaciones que se pueden entablar entre organizaciones criminales y terroristas son más ocasionales e interesadas (parasitarias) que alianzas estratégicas (Passas, 2007).

\section{Contrabando de oro y piedras preciosas}

Sobre esta cuestión deberíamos hacer unas cuantas precisiones. Muchos autores ${ }^{16}$ afirman que al Qaida está usando el mercado negro de oro y piedras preciosas para conseguir fondos para sus células, esconder dinero amenazado por sanciones financieras, blanquear beneficios de actividades delictivas, convertir el metálico en objetos que mantienen su valor y son fácilmente transportables (Escobar Stemman, 2004). Sin embargo, desde esta investigación no se ha podido encontrar evidencias significativas de la existencia de financiación proveniente del comercio ilegal de oro y diamantes (Passas, 2004; Passas and Jones, 2006). Esta falta de consistencia también se menciona en el informe de la comisión de investigación del 11 de septiembre donde se afirma que «no existen evidencias de que al Qaida se haya financiado con el mercado negro de diamantes africano» ${ }^{17}$. Otra cosa es que se hayan dado casos en los que personas asociadas a grupos terroristas hayan hecho transacciones en el mercado de diamantes y piedras preciosas, que sí se han demostrado evidencias de ello ${ }^{18}$.

${ }^{16}$ Esta asociación entre al Qaida y el mercado de diamantes y piedras preciosas se ha confirmado por la mayoría de autores de la literatura pero proviene fundamentalmente del trabajo de cinco autores que luego se ha ido citado por otros autores y que, por la fuerza de la repetición, se ha convertido en cierta. Artículos en el Washington Post de Doug Farah, Wall Street Journal, un documental de la BBC, un informe de la ONG Global witness (2003) e informes públicos procedentes de un tribunal especial en Sierra Leona han sido las principales fuentes de dicha información y, en ningún caso, aportan fuentes de información propias sino que se citan mutuamente. Respecto a esto, la investigación realizada no puede confirmar esta teoría ya que carece de evidencia empírica y adolece de exageraciones y asunciones sin fundamento. Hemos realizado entrevistas a miembros de personal de inteligencia e investigadores de Naciones Unidas, Estados Unidos y Europa, a oficiales de ONGs, a periodistas, empresarios y profesores universitarios que han estudiado la industria del diamante en los últimos cinco años. También se ha procedido a una revisión de informes públicos y confidenciales del atentado a la embajada de Oeste de África y no se ha podido encontrar evidencia empírica que sustente dicha relación.

17 Final Report. National Commission on Terrorist Attacks upon the United States (2004: 171).

${ }_{18}$ De la revisión de los documentos judiciales sobre el juicio del ataque a la Embajada de Estados Unidos en África se desprende que determinadas personas procu- 
Además de la obtención de fondos, las organizaciones terroristas necesitan mover el dinero a lo largo del territorio nacional e internacional, dejando el menor rastro posible. Diferentes modos de transferencia se han identificado como los más usados por grupos relacionados o inspirados por al Qaida, a continuación explicamos lo más usuales y su funcionamiento.

\section{Modos de transferencia de fondos}

Los métodos para transferir fondos utilizados por los grupos terroristas relacionados con al Qaida son fundamentalmente tres: en primer lugar, al igual que cualquier ciudadano común, el uso del sistema financiero formal. En segundo lugar, los sistemas de transferencias de fondos informales como el sistema denominado Hawala. Y, en tercer lugar, el medio de transferencia más antiguo, los correos personales.

Respecto al uso del sistema financiero tradicional, debemos decir que, como se ha puesto en evidencia a partir de las investigaciones del 11 de septiembre y otras investigaciones llevadas a cabo también en España sobre grupos inspirados o relacionados con al Qaida, el uso del sistema financiero formal por parte de los miembros de estos grupos no presenta ninguna característica especial o particular que lo haga distinto del uso que podría realizar cualquier otro ciudadano. También el hecho de que las cantidades transferidas de banco a banco suelan ser siempre de escasa importancia ${ }^{19}$, hace que pasen desapercibidas en el volumen de dinero que se maneja diariamente en el sistema financiero, sobre todo antes del 11 de septiembre que los controles estaban orientados a prevenir el blanqueo de capitales y lo que buscaban eran grandes volúmenes de dinero que se querían introducir en el sistema financiero. Otro motivo por el cual dichas transacciones no se detectan fácilmente es que, habitualmente, los miembros de las células tienen una actividad legal que les permite realizar dichos movimientos de capital sin necesidad de levantar sospechas. En definitiva, los movimientos de capital que realizan los

raron financiación procedente del mercado de diamantes, del contrabando de especies protegidas y del contrabando de diversos productos como: plátanos, insecticidas, cuero, limones, aceite de palma, calculadoras, camellos, coches, cemento, tractores y un largo etcétera (Passas, 2007).

19 En el caso del 11 septiembre y según el informe de la Comisión de investigación del 11de septiembre, las transferencias que se hicieron a Estados Unidos no superaron los 70.000 dólares. En el caso de la operación dátil, las transferencias fueron mucho menores, como máximo 15.000 dólares. 
terroristas no son distintos al de un ciudadano normal, no tienen un conocimiento especialmente destacable del funcionamiento del sistema financiero, con lo cual es muy difícil que puedan detectarse transacciones destinadas a financiar actividades de apoyo o de soporte a terroristas.

Los sistemas informales de transferencia de fondos sí merecen una atención especial ya que parecía que, después del atentado del $11 \mathrm{~S}$, iban a aumentar con el incremento del control ejercido sobre el sistema financiero ${ }^{20}$. Estos sistemas se refieren a cualquier red o mecanismo que sirve para transferir fondos o valores de un lugar a otro sin dejar un rastro formal de la transacción o sin pasar por instituciones financieras formales (Passas, 2002). En este punto hay que hacer una distinción conceptual pero útil en la práctica para distinguir dos tipos de sistemas informales de transferencia de fondos. Por un lado, están los sistemas informales de transferencia de fondos $\mathrm{y}$, por otro, los métodos informales de transferencias de fondos. Los primeros constituyen un sistema étnico y tradicional de transferencia de valores y fondos que tienen su origen en India y China pero que con la inmigración y la globalización se han extendido a otros lugares. Los segundos, se refieren a pequeños grupos, que no redes, que emplean métodos informales de transferencia de fondos y valores generalmente ilegales (Passas, 2006c: 48) ${ }^{21}$.

El sistema informal de transferencia de fondos más conocido, también llamado "sistema bancario clandestino» es el Hawala. Hawala significa transferencia en árabe y se basa en transferir dinero o valores de un lugar a otro. Originariamente se utilizaba de forma

${ }^{20}$ En el informe final de la comisión de investigación de los ataques del 11 de septiembre (National Commission on Terrorist Attacks upon the United States), 2004: 1819 se alertaba del posible aumento de estos sistemas como consecuencia del ataque del 11 de septiembre. Se alertaba del incremento del uso de sistemas informales de transferencia de fondos así como un mayor recurso a la delincuencia como modo de financiación de los grupos terroristas, para así evitar ser controlados y detectados por el refuerzo de los controles impuestos en el sistema financiero local e internacional. Sin embargo, esta afirmación no se ha constatado en la práctica ni se ha visto un incremento real en los grupos desarticulados por la policía en operaciones posteriores.

${ }^{21}$ Estos métodos informales no requieren una red muy extendida de personas, bastan dos individuos para llevar a cabo las transferencias; se utiliza el sistema financiero formal pero intentando no dejar rastro o camuflando los fondos ilegales con transacciones legales o aparentemente legales; normalmente tienen una finalidad delictiva o se combina con otros delitos; y tienen capacidad para mover grandes cantidades de dinero, con lo que no sólo es utilizado por los terroristas sino también por otro tipo de delincuentes (Passas, 2006c: 49). Ejemplos de este tipo de métodos son: transferencias en especies, manipulación de facturas, desvío de dinero en el comercio, uso ilícito de tarjetas de crédito o débito, pagos a través de Internet, etc. 
local en China y otras regiones del Asia oriental en las que todavía se siguen utilizando estos sistemas con diferentes nombres: Fei-Chi'ien (China), Padala (Filipinas), Hundi (India), Hui Kuan (Hong Kong) y Pei Kwan (Tailandia). Es un sistema de transferencia de dinero basado en la confianza entre emisor y destinatario en una tercera persona-operador de hawala- que es el que actúa de intermediario ante la ausencia de un sistema bancario formal. Este sistema se desarrolló también con mucha fuerza en Afganistán tras el conflicto soviéti$\mathrm{CO}^{22}$. Además del servicio de transferencia de fondos, estos agentes de cambio proporcionan una amplia gama de servicios financieros y no financieros tanto locales, nacionales como internacionales. Entre las actividades financieras que realizan, además de las transferencias de fondos, destacan servicios como cambio de moneda, microfinanciación, custodia de depósitos, etc. Entre las actividades no financieras ofrecidas se incluyen servicios de Internet, fax, teléfono, asistencia comercial nacional e internacional, etc. (Maimbo, 2003: 8).

Para comprender cómo funciona este sistema debemos entender que existen dos relaciones importantes en este tipo de transacciones. Por un lado, la relación entre el operador de hawala de un determinado lugar (país A) y su cliente, quien ordena al primero una determinada transacción o envío de dinero a un destinatario que se encuentra en otro lugar (país B). El operador de hawala o intermediario del país A establece una relación con el operador de hawala del país B para que éste último entregue el dinero al destinatario final del dinero, cancelando así el pago. El gráfico a continuación explica las dos relaciones.

La segunda relación importante en este sistema es la relación de ajuste de cuentas que se produce entre los operadores de hawala de los dos lugares. Cada vez que un operador de hawala realiza una orden de pago, se crea una deuda informal entre los operadores de hawala que luego saldarán entre ellos. Para que este sistema Hawala funcione debe existir una caja importante dinero en los polos de la

${ }^{22}$ En el caso de Afganistán, después de 20 años de conflicto en el país, el sector financiero formal estaba prácticamente inoperativo. Un marco legal y regulador anticuado, un personal con falta de preparación técnica y de gestión, la inexistencia de sistemas informáticos aplicados a los bancos, redes de telecomunicaciones precarias eran algunos de los problemas a los que se enfrentaba el sistema bancario formal. Como consecuencia de esta situación, se empezó a desarrollar un mercado informal que empezó a cubrir las necesidades financieras de la población. Estos operadores de hawala o agentes de cambio de moneda empezaron a dar un servicio mejor organizado, más eficiente y más barato de transferencia de fondos a nivel local e internacional. Se calcula que hay entre 500 y 2000 personas dedicadas a esta actividad en Afganistán (Maimbo, 2003: 5). 
transacción. Así, el operador de hawala del país B puede dar el dinero ordenado por el emisor del país A al destinatario del país B. Para este tipo de transacción, los operadores de hawala usan el dinero que reciben de sus clientes y de las actividades realizadas para llevar a cabo las órdenes de transferencia que reciben de parte de otros operadores de hawala. Los fondos que frecuentemente engrosan las cajas de estos intermediarios proceden de: envíos de dinero de expatriados a sus familias, pagos por importaciones, fondos de inversión, servicios realizados desde otros países pero que deben pagarse localmente, evasión de impuestos, contribución a militantes terroristas, dinero procedente del trafico de drogas y de personas, etc. (Passas 2002).

\section{Fig. 1: Passas (2003a: 5)}

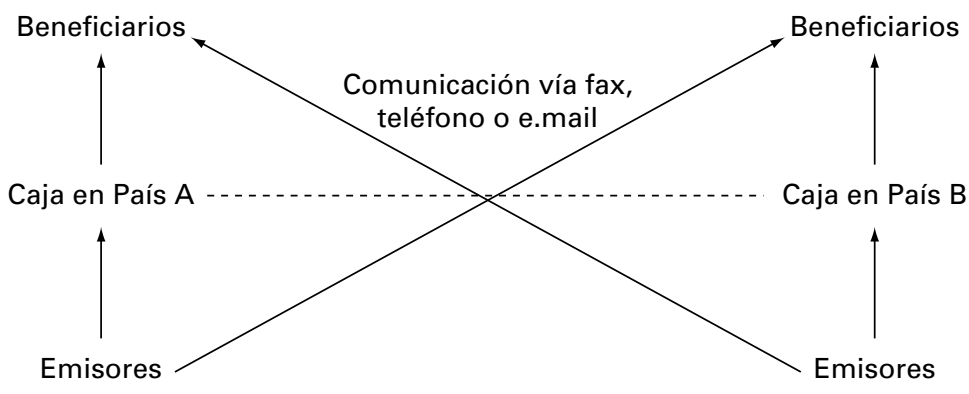

El atractivo del hawala reside en la confianza que genera en sus usuarios, la rapidez de sus transacciones, el anonimato, la ausencia de imposición fiscal, el bajo coste y la escasa burocracia requerida comparativamente con el sistema financiero formal. Respecto al coste, éste suele oscilar entre el 1 y $2 \%$ del volumen de la transacción, no existiendo, en principio, un volumen máximo de fondos por transferencia. El anonimato es asegurado gracias a la ausencia de un sistema de control y seguimiento de las transacciones, común a los sistemas informales de transferencias de fondos y la inexistencia de requisitos formales exigibles en su funcionamiento. El sistema de registro y control de las transacciones es individual y depende de la forma de gestión y trabajo de cada uno de los operadores de hawala. Tampoco existe una documentación común a todos ellos a la hora de registrar a los clientes, a lo sumo se guardan unos códigos o registros que dependerán de la forma de trabajo de cada agente y de su forma de llevar y gestionar el negocio. Sin embargo, este sistema, aunque no asegura la transparencia no quiere decir que no se lleve con el control adecuado y que no 
sea posible la trazabilidad del dinero. Existe la posibilidad de seguir el rastro del dinero entre emisor y receptor y, en cada uno de los lados, existe información sobre las transacciones, clientes, etc.

Por otro lado, este sistema suele funcionar combinado con el uso del sistema financiero formal y tradicional. Los operadores de hawala suelen usar los dos sistemas en cada transacción pero la ventaja para destinatarios y emisores radica en que no es necesario que ellos mismos abran una cuenta bancaria, son los operadores de hawala que operan a través de sus cuentas abiertas en varios países ${ }^{23}$. Por otro lado, para arreglar las cuentas entre los operadores de hawala, usan en muchas ocasiones el sistema financiero formal. Cuando las cuentas no pueden saldarse con las transacciones recíprocas, se utiliza el sistema financiero formal para realizar transferencias a sus respectivas cuentas bancarias (Maimbo, 2003: 16, Passas, 2003c).

A parte del hawala también existen otros sistemas informales de transferencia de fondos que operan de forma similar y que son utilizados por la población de forma legítima sin dejar rastro de dichas transacciones:

- Hundi: es distinto al hawala aunque en Pakistán y Bangladesh el término utilizado para el hawala es «hundi». Hundi es uno de los instrumentos de crédito más importantes de la India, parecido a las prácticas financieras europeas de los siglos XII y XIII (Passas, 2002).

- Mercado negro del peso y otras redes de cambio.

- Fei chien y otras variantes asiáticas.

Todos estos sistemas están activos en muchos países y constituyen una forma muy utilizada de pago para todos aquellos que deseen realizar transferencias, tanto legales como ilegales. Todas las características que lo hacen atractivo para la población general, su eficacia, la posibilidad de acceso a lugares remotos y subdesarrollados, el anonimato que garantiza, su bajo coste, etc. también son características apreciadas y útiles para aquellos que desean mandar fondos ilegales o destinados a acciones terroristas. Sin embargo, es un sistema que sobrevive gracias a la confianza de la población y el hecho de que, en determinados casos, sea utilizado ilegítimamente no lo convierte en perverso o en meritorio de su erradicación. Es más, su supresión o prohibición sin más, constituiría un grave perjuicio para la población a la que sirve. Sería conveniente que cualquier forma de

${ }^{23}$ Final Report. National Commission on Terrorist Attacks upon the United States, 2004: 25 . 
control o regulación se fundamentara en una comprensión profunda del sistema, evitara daños colaterales y consiguiera el consenso e implicación tanto de operadores como de usuarios en su regulación y formas de cumplimiento de la misma (Passas, 2003a). Los actuales medios de regulación, aun basados en sus propios estándares, no están cosechando demasiados éxitos (Passas, 2005).

Por último, el método más antiguo de transferencia de fondos que se sigue utilizando regularmente por grupos terroristas es el correo personal. Al Qaida y otras organizaciones terroristas han usado tradicionalmente correos personales tanto para transportar dinero como información, mercancías, etc. Las personas reclutadas por al Qaida para estas tareas suele tener bajo perfil ${ }^{24}$, gozar de su más absoluta confianza (familiares, esposos, novios, etc.) y, normalmente, desconocen el destino y uso que se va a hacer de la mercancía o dinero que transportan. Tenemos numerosos ejemplos en España y en el extranjero de transferencias de dinero o mercancías realizadas a través de correos: ej. $11 \mathrm{~S}$, Operación dátil, $11 \mathrm{M}$. El hecho de que se utilicen estos correos tiene consecuencias tanto positivas como negativas desde el punto de vista de su control. Por un lado, permiten el transporte de valores y bienes sin dejar rastro pero, por otro, la utilización de estos métodos requiere un esfuerzo en planificación, comunicación y diseño de estrategias que también ofrece oportunidades a la policía para su detección ${ }^{25}$.

\section{Financiación del 11S y del 11 M}

En este apartado queremos comparar dos atentados muy distintos respecto a su preparación, ejecución y financiación. Estas diferencias ponen también en evidencia cambios en las formas de organización de los grupos relacionados con al Qaida y la emergencia de nuevos grupos con mayor o menor vinculación con los miembros de al Qaida, donde la descentralización es cada vez más importante y en los que la relación con el núcleo de al Qaida no es ni necesaria para poder conseguir sus objetivos. A continuación describimos la financiación de ambos atentados.

El 11 septiembre fue una operación especial en cuanto a su envergadura e impacto, para ello fue preparada cuidadosamente y fi-

${ }^{24}$ Ibid., Según dicho informe el bajo perfil puede originarse por su situación personal, habilidades comunicativas, etnia, documentación, etc.

${ }^{25}$ Final Report. National Commission on Terrorist Attacks upon the United States, 2004: 27. 
nanciada en todos los aspectos por al Qaida. Esta organización financió no solo el ataque sino la preparación de los suicidas para el mismo, el alojamiento, la manutención, el entrenamiento como pilotos, etc. Alrededor de 300.000 dólares fueron depositados en cuentas bancarias de Estados Unidos llegando por tres vías diferentes: la primera de ellas fueron transferencias de banco a banco desde fuera de Estados unidos (Emiratos Árabes y Alemania) realizadas por dos intermediarios: Ali Abdul Aziz Ali (Ali) ${ }^{26}$ y Mustafa al Hawsawi. El primero usó la cobertura de su actividad legal como mayorista de informática y distintos alias pudiendo así hacer las transacciones sin levantar ninguna sospecha. La mayoría de dinero enviado no procedía de sus cuentas sino de sacas de dinero en metálico que tenía en su casa. Otras transferencias fueron hechas desde Alemania por Binalshibh. La segunda fuente de financiación vino a través del uso de tarjetas de crédito vinculadas a bancos extranjeros y la tercera se produjo gracias al transporte físico de dinero y cheques de viaje realizado por los propios protagonistas del atentado, con el que abrieron distintas cuentas en Estados Unidos.

En la financiación del atentado del 11 de septiembre no se ha constatado la ayuda de ciudadanos americanos, ni de carácter logístico ni financiero, ni tampoco la utilización del hawala como método de transferencia de fondos. Las transferencias de dinero no fueron muy elevadas, la más importante fue de 70.000 dólares con lo cual los sistemas puestos en marcha para detectar irregularidades en el sistema financiero (entonces vinculados al blanqueo de capitales) no estaban preparados para este tipo de transacciones. Por otro lado, es llamativo el hecho de que los miembros del comando que perpetró el ataque devolvieran el dinero que les sobró (36.098 dólares) a través de bancos y de la empresa Western Union. Este hecho nos adelanta una de las diferencias fundamentales entre la financiación del terrorismo y delincuencia organizada. Las redes de delincuencia organizada siempre persiguen el ánimo de lucro y sus actividades van siempre encaminadas a producir el máximo de beneficios. Sin embargo, el terrorismo tiene objetivos alejados del lucro económico: la financiación y el dinero son su herramienta principal pero no un fin en si mismo.

Sobre el atentado del 11 de Marzo todavía quedan algunas incógnitas por desvelar, sobre todo respecto a la autoría intelectual de los atentados o la coordinación de los mismos. Sin embargo y en referencia a la célula operativa que ejecutó el atentado en Madrid, se

${ }^{26}$ Ali transfirió un total de 119.500 dólares en seis transacciones desde abril a septiembre de 2000 . 
llevó a cabo por un grupo reclutado entre los círculos de la delincuencia común, especialmente el tráfico de drogas y sustracción de vehículos en el que Jamal Ahmidan tenía un papel preponderante ${ }^{27}$. En consecuencia, el grupo operativo se componía de personas integradas en redes delincuenciales comunes (relacionadas a nivel nacional e internacional) que se transformaron en directos implicados en actuaciones terroristas ${ }^{28}$.

En lo que afecta a la financiación del atentado, el ataque se autofinanció enteramente por el grupo implicado a través de medios legales e ilegales, principalmente a través de fondos procedentes del tráfico de drogas y de la sustracción de vehículos a motor ${ }^{29}$. En relación con los medios económicos procedentes de actividades ilegales, los fondos del tráfico de hachís fueron los responsables de la adquisición de $210 \mathrm{~kg}$. de explosivos y 260 detonadores que luego volaron lo trenes en Atocha. La hipótesis más probable es que se realizara un trueque entre Jamal Ahmidan y José Emilio Suarez Trashorras en base a una deuda que tenía éste último con el primero por ser su proveedor habitual de hachís. José Emilio tenía un impago de entre 25 y 30 kilos de hachís y probablemente le consiguió los explosivos a cambio de saldar una parte de dicha deuda ${ }^{30}$. Jamal Ahmidan tenía como forma habitual de vida el tráfico de hachís y la falsificación de documentación.

Otra parte importante de la financiación procedió de fondos legales provenientes de la actividad laboral de los integrantes del grupo, especialmente de Jamal Ahmidan cuyo protagonismo en la financiación, organización y planificación del atentado fue fundamental. La mayoría de los miembros del grupo realizaban actividades laborales de escasa cualificación y de forma temporal. Destaca la actividad en sectores de servicios (hostelería, seguridad, etc) y como autónomos, regentando locutorios o tiendas de venta y reparación de teléfonos móviles, diseño y colocación de rótulos y venta de vehículos usados. Los fondos obtenidos por estas actividades, especialmente la de Jamal

27 Auto de prisión del Juzgado Central de instrucción número 6. Sumario 20740. 18 de Julio de 2004, p. 33.

28 Ibid., p. 28.

29 No hay constancia de ninguna transferencia o movimiento de fondos procedente del exterior. Auto de procesamiento. Sumario 20/40. Juzgado Central de Instrucción número 6 de la Audiencia Nacional, p. 1138.

${ }^{30}$ Informe de la Comisaría General de Información incluido en el Auto de procesamiento del $11 \mathrm{M}$, disponible en la página web: http: //www.elmundo.es/documentos/2006/04/11/autohtml/index.html?cual=1126. El importe de la deuda podría ascender a 45.000 euros aproximadamente, teniendo en cuenta que el kilogramo de hachís podría valorarse entre 1.275 y 1.500 euros (p. 1133). 
Ahmidan, permitieron el alquiler de los inmuebles que sirvieron para el alojamiento y preparación de los atentados: finca de Chinchón (5.421 euros), Chalet en Albolote (800 euros) y el piso de Leganés (1.800 euros) $)^{31}$. Otros miembros de la célula obtenían ingresos procedentes del desempleo o de prestaciones sociales. Por último, también los fondos procedentes de la economía sumergida constituyeron una fuente importante de financiación de la manutención de los miembros de la célula. En general, la situación económica de todos los miembros era del todo precaria, siendo incapaces de afrontar los gastos de manutención y alojamiento en su vida diaria, demostrándose este punto por los continuos descubiertos bancarios y las escasas transferencias realizadas ${ }^{32}$.

El coste total del atentado se estima en alrededor de 100.000 euros si incluimos dentro de este precio los costes de adquisición del material explosivo (entre 31.835 y 45.000 euros), los inmuebles arrendados y las tarjetas SIM y móviles adquiridos (54.271 euros), sin contar el coste de las armas y de la documentación falsa. Sin embargo, el grupo disponía en droga de una cantidad mucho mayor. En un registro de la casa de los primos de Jamal Ahmidan se incautó droga por valor de entre 1.353.677 y 1.537 .663 euros $^{33}$.

Las transferencias y movimientos de fondos en las cuentas investigadas son todas muy pequeñas, no ascendiendo la más importante a más de 10.000 euros. Hay que destacar un caso de utilización del hawala por parte de la hermana de Rachid Oulad Akcha que pagó 10.600 euros a la empresa Hispano-alemana de Motores y Repuestos, SL. para que se enviara unas mercancías a un cliente en Tetuán. Luego este compensaría los pagos en Marruecos ${ }^{34}$.

Los modos de transporte para el envío de explosivos utilizados fueron correos personales. Se hicieron tres viajes de Asturias a Madrid llevando explosivos y dinero en metálico. Cuando Trashorras consiguió los explosivos organizó el transporte utilizando correos: chicos jóvenes que no conocían ni el contenido de la mercancía ni el fin de la misma. El primer viaje fue realizado por Sergi Alvarez Sanchez que fue encargado de llevar una bolsa en un autobús a Madrid a cambio de 600 euros, pensando que eran CDs ilegales. El segundo via-

${ }^{31}$ Auto de procesamiento. Sumario 20/40. Juzgado Central de Instrucción número 6 de la Audiencia Nacional, p. 1129.

32 Ibid., p. 1129.

33 Ibid., p. 1137. Sin embargo, pese a la breve descripción que se realiza en el Auto, parece que responde más a un método informal de transferencia de fondos que a un ejemplo de hawala.

34 Ibid., p. 1133. 
je fue realizado por Gabriel Montoya Vidal, menor de edad, que viajó en las mismas circunstancias. El tercer viaje lo hizo Antonio Ivan Reis Palacio que debía dar también 3000 euros a Jamal Ahmidan pero éste nunca recibió el dinero. El cuarto viaje fue realizado personalmente por Jamal Ahmidan acompañado de Mohamed Oulad y Abdenabi Kounjaa que hicieron el viaje para recoger más explosivos.

Estos ejemplos que hemos descrito nos sirven para conocer un poco más sobre la mecánica y modus operandi de la financiación de dos actos muy distintos. La descripción de los atentados de Madrid contrasta con el modus operandi de los atentados del 11 de septiembre. El atentado de EEUU fue financiado y organizado por al Qaida y en el caso del $11 \mathrm{M}$ se trata de grupos inspirados en la ideología de al Qaida, de carácter autónomo donde la financiación y la organización es puramente local.

\section{Control de la financiación del terrorismo: elementos para el debate}

Después de los atentados del $11 \mathrm{~S}$, importantes medidas se han puesto en marcha para detectar e impedir la financiación de actos terroristas, reducir el apoyo económico de sus miembros y simpatizantes, detectando fondos sospechosos y procediendo a su bloqueo. Se han introducido nuevos controles para que los Estados puedan identificar la fuente y el destino de los fondos ${ }^{35}$, se han creado grupos de acción para investigar la financiación del terrorismo ${ }^{36}$, se ha promovido la co-

${ }^{35}$ La respuesta oficial a las actividades terroristas ha sido nacional e internacional. La ley americana denominada Patriot Act (2001) ha endurecido la regulación contra el blanqueo de dinero y ha otorgado a la policía amplios métodos y poderes para la prevención e investigación de actividades terroristas en Estados Unidos. La Executive Order 13224 bloqueó propiedades americanas y bloqueó todas las transacciones de sujetos u organizaciones que apoyaran actividades terroristas. La iniciativas internacionales más importantes han sido: la Convención de Naciones Unidas de 1999 sobre financiación del terrorismo, la Resolución 1267 del Consejo de Seguridad de Naciones Unidas de 1999 estableciendo un comité de sanciones para controlar y aplicar las sanciones financieras al régimen Talibán, la resolución del Consejo de Seguridad de Naciones Unidas 1373 (2001) sobre financiación del terrorismo y las nueve recomendaciones especiales sobre financiación del terrorismo del Financial Action Task Force (FATF) (ver también la Resolución del Consejo de Seguridad de Naciones Unidas 1390 [2002]).

${ }^{36}$ LexisNexis, "US and Saudis Act to Freeze Charity's Assets», The New York Times, 23 de Enero, 2004: LexisNexis, Tom Godfrey, «Canadian Assets frozen, RCMP Sweep in on $\$ 2$ Million Thought connected to Al Qaida Terrorist Network», The Ottawa Sun, 8 de Noviembre, 2001. 
operación internacional entre los países, se han promulgado nuevas normas para criminalizar la financiación del terrorismo ${ }^{37}$, se han promovido medidas para incrementar la transparencia en el sector financiero e identificar las operaciones sospechosas ${ }^{38}$.

El éxito y los resultados de estas medidas se han evaluado en base a la cantidad de cuentas bloqueadas o la cantidad de casos sospechosos analizados ${ }^{39}$. Según estos indicadores, podemos considerar que la labor de los controles de las instituciones financieras ha sido satisfactoria, aunque sea difícil determinar con estas medidas cuántos ataques terroristas se han prevenido gracias a estas actuaciones o cuánto dinero destinado a financiar estructuras terroristas se ha eliminado del circuito. Sin embargo, estos controles no han estado exentos de problemas y de aplicaciones irregulares. Tal como la comisión de investigación del 11 de septiembre ha puesto de manifiesto, en muchos casos, el bloqueo de determinados fondos sospechosos ha sido prematuro y problemático a posteriori ${ }^{40}$. En algunos casos, no ha habido suficientes pruebas en la investigación posterior para justificar las acusaciones. Además, aunque respecto al sistema financiero podamos estar satisfechos de estos resultados, las organizaciones benéficas y los sistemas informales de transferencia de fondos son difíciles de regular y pueden todavía ser una fuente de ingresos importante para los terroristas ${ }^{41}$.

Es necesario profundizar aún más en el conocimiento del fenómeno al que nos estamos enfrentando para idear y aplicar medidas de control y prevención más adecuadas a la realidad, intentando evitar

37 Ver Naciones Unidas.

38 "UK Minister Calls For Less «Money Secrecy» to Combat Terrorism Crime», Londres FCO, 15 de Octubre, 2002.; LexNexis, Jason Bennetto, «UK police freeze $\$ 70$ m in Taliban money», Hamilton Spectator (Ontario, Canada), 4 de Octubre, 2001; LexisNexis, "Erika Kinetz, "Caribbean weighs costs, Offshore centres adjust to new OECD rules», The International Herald Tribune,16 de Mayo, 2002.

39 «UK Minister Calls For Less Money Secrecy to Combat Terrorism, Crime, London FCO, 15 de Octubre de 2002.

${ }_{40}$ Ver los casos de Barakaat y alguna organización benéfica que se relatan en el Staff Monograph on Terrorist finance (National Commission on Terrorist Attacks upon the United States, 2004). Ver también Lee (2002) y la página web de la UK Charity, comisión donde se describen los resultados de sus investigaciones. En el caso de Palestinians Relief and Development Fund, conocido como Interpal, una organización considerada de terrorismo global fue acusada de apoyar actividades políticas y violentas de militantes de Hamas. La comisión considera que las autoridades americanas no fueron capaces de aportar pruebas que apoyaran dicha acusación. Como consecuencia de ello, las cuentas del Banco Interpal fueron desbloqueadas y el caso sobreseido en septiembre de 2003 (http: //www.charity-commission.gov.uk/investigations/inquiry reports/interpal.asp). Ver también Passas (2006a) y Passas (2006b).

${ }^{41}$ LexisNexis, "Follow the money», The Economist, 1 de junio, 2002. 
efectos contraproducentes o daños colaterales innecesarios. Hasta ahora se ha estado luchando contra un fenómeno sin saber muy bien en qué consistía y aplicando medidas indiscriminadas sin medir los daños y consecuencias generadas. Para conocer un poco más las características del mismo analizaremos algunos puntos que debemos tener en cuenta si intentamos controlar la financiación del terrorismo.

\section{La naturaleza y organización social de grupos terroristas}

Uno de los aspectos más importantes a tener en cuenta a la hora de saber dónde poner el énfasis respecto al control de la financiación, es la organización y naturaleza de estos grupos terroristas. Dependiendo de la organización, la centralización de sus funciones, la distribución de tareas entre los miembros, la deslocalización o localización de las actividades, etc., necesitaremos incidir en unos aspectos u otros a la hora de controlar las fuentes económicas de estos grupos. Existen todavía distintos enfoques, algunos radicalmente distintos, sobre la naturaleza y organización de estos grupos, con la consiguiente implicación en las recomendaciones sobre medidas de lucha contra el mismo ${ }^{42}$. La única manera de saber si las medidas de control y regulación que estamos poniendo en marcha son compatibles o contradictorias es ahondando en la descripción correcta de estas redes, su jerarquía, las formas en que se relacionan y comunican, el modo en que se está llevando a cabo la financiación local e internacional, etc.

Para ser más concretos, si tenemos en cuenta que al Qaida no es una organización sino una idea o marco de referencia que inspira de forma local la operatividad individual y de varios grupos, la cantidad de dinero que nosotros debemos controlar es mucho más pequeña que si vemos el fenómeno desde su perspectiva global e internacional, los medios utilizados para generar financiación dependen de las condiciones locales y la delincuencia común será utilizada de forma regular y permanente, existiendo menor necesidad de acudir a transferencias de fondos y a la comunicación entre grupos. Por el contrario, las medidas de lucha contra la financiación del terrorismo impuestas por los Estados han estado hasta ahora inspiradas en el fenómeno del blanqueo de capitales, identificando grandes flujos monetarios a nivel transnacional, distintos a los que hemos visto que se realizan a nivel local (ej $11 \mathrm{M})$ para financiar las actividades terroristas de grupos relacionados o inspirados por al Qaida.

42 Ver la versión de Gunaratna (2002) y Burke (2002). 
En suma, es fundamental conocer y profundizar sobre la organización de organizaciones terroristas, según la dimensión local o internacional que tengan, según la complejidad y las relaciones internacionales que cuenten será el abordaje y su control. Los controles internacionales funcionarán si la organización de al Qaida es internacional y su funcionamiento centralizado, si por el contrario la descentralización es la norma, medidas de carácter local serán mucho más importantes (Passas, 2007).

\section{Carácter local de las actividades de financiación}

Íntimamente ligado al punto anterior está el aspecto de la localización de las actividades terroristas, incluyendo dentro de ellas labores como el reclutamiento de miembros terroristas, el proselitismo y las labores de radicalización, en un paso más adelante, la organización de atentados, el abastecimiento de material para los mismos, la financiación de los mismos. Todas estas actividades requieren una organización pero se sirven de los recursos locales para desarrollarse. Todas estas actividades están determinadas por factores locales como: la comunidad de referencia, las redes de delincuencia o actividades ilegales más enraizadas en el lugar, la solidaridad de los miembros de la comunidad, los factores sociales locales, las políticas de inmigración, la situación de las cárceles, etc. Todas estas condiciones ayudan y alimentan el nacimiento y desarrollo de grupos que encuentran los instrumentos necesarios para ejercer sus actividades. Es entonces a nivel local donde también tendremos que extremar las precauciones y tendremos que luchar para que las condiciones dificulten la instalación y desarrollo de grupos terroristas.

En lo que afecta a la financiación, debemos mejorar el conocimiento local del problema, las relaciones entre financiación del terrorismo y delincuencia común, los modos de transferencia de fondos utilizados, los mecanismos de impedir la trazabilidad llevados a cabo por estos grupos, etc. Un conocimiento actualizado de estos métodos nos conducirá a una mejora de la prevención y detección de esos casos. Por otro lado, la cooperación internacional y la actuación a gran escala en la financiación del terrorismo es importante pero, en muchos casos, la actuación con un enfoque global no abarca ni controla la financiación que se produce a nivel local, pasando ésta desapercibida. 


\section{Coste de los atentados y necesidad de fondos}

Una de las cuestiones que más llama la atención al estudiar aspectos de la financiación del terrorismo, es el escaso coste que suponen los actos terroristas y, en especial, los actos perpetrados por el terrorismo de al Qaida, que tantas víctimas y daños colaterales está provocando. En el caso del 11 de septiembre, el informe de la comisión de investigación de los atentados calculó su coste entre 400.000 y 500.000 dólares en dos años. La mayoría de este dinero ya hemos visto que fue proporcionado por al Qaida, a través de transferencias hechas a Estados Unidos que pasaron desapercibidas bajo unos sistemas de control que no estaban preparados para detectar este tipo de transacciones ${ }^{43}$.

Otros ejemplos de ataques terroristas en los que se ha calculado el coste nos muestran la escasa financiación que requiere su preparación y ejecución comparativamente con el daño que provocan estos atentados de gran magnitud:

El primer atentado del World Trade Centre costó menos de 19.000 dólares

- Atentado de Bali se calcula que costó 20.000 dólares.

- Atentado en Kenya y Tanzania: 50,000 dólares.

- Estambul: 40,000 dólares.

- 11 Septiembre, entre 400,000-500,000 por un periodo de dos años.

- 11 Marzo: alrededor de $100.000^{44}$.

- Londres: algunos cientos de libras (2006a).

Sin embargo, esto se refiere al coste de determinadas operaciones, sin tener en cuenta gastos como la financiación de una organización, sus miembros, actividades cotidianas de entrenamiento, captación, adoctrinamiento, manutención, etc.

\section{Cómo reducir el apoyo y seguimiento de simpatizantes}

Las medidas a corto plazo deben también combinarse con medidas a largo plazo que ataquen las causas y raíces fundamentales del terrorismo. Tenemos en España un ejemplo en la lucha contra el te-

43 Anexo A. Final Report. National Commission on Terrorist Attacks upon the United States, 2004: 131.

${ }^{44} \mathrm{Si}$ incluimos únicamente los explosivos el coste supuso alrededor de 50.000 euros. 
rrorismo de ETA. Un elemento fundamental que ayudado al declive y merma de actividad de la organización terrorista ha sido el ataque al entorno social y al movimiento de apoyo que suponía el entorno de ETA. Es por este motivo que es imprescindible entender las razones de emergencia de la militancia en estos grupos, porqué continúan con la lucha y qué factores apoyan tanto la radicalización como el desistimiento en este tipo de organizaciones. Sólo así podremos idear medidas de reducción del apoyo social y de emergencia de nuevos simpatizantes o militantes. Asimismo, en este contexto también deberíamos ahondar sobre las consecuencias que tienen los controles financieros para la militancia y si efectivamente producen los efectos deseados.

Tampoco podemos pensar en medidas de control de la financiación sin situarnos en el contexto étnico y religioso de estos grupos, intentando que la visión y mentalidad occidental provoque mayores riesgos. Sin este esfuerzo de empatía no sólo conseguiremos que las medidas impuestas sean contraproducentes sino que produzcan daños colaterales más importantes de los que se pretenden evitar. Como hemos visto en el apartado dedicado al Hawala, las medidas agresivas de erradicación de sistemas que sirven a una población al completo pueden ser no sólo negativas y extralimitadas, también contraproducentes, consiguiendo el efecto contrario: incrementar el odio y dotar de más argumentos de apoyo y exaltación del extremismo.

Asimismo, las políticas y prácticas internacionales también puede tener efectos contraproducentes y multiplicar los daños colaterales. En este momento, la guerra de Irak está siendo lo que fue antaño Afganistán, un campo de entrenamiento para militantes y también una forma de generar motivos y oportunidades para el extremismo. Este efecto llamada no sólo puede aumentar el número de simpatizantes individuales sino también aumentar en número de empresas o negocios gestionadas por estos individuos que pueden contribuir y colaborar en la financiación de actividades terroristas.

\section{Mejora de la transparencia y trazabilidad}

Importantes y cuantiosos esfuerzos se están llevando a cabo para mejorar la transparencia del sistema financiero y conseguir mejorar el seguimiento y la trazabilidad del dinero. Hasta ahora, las medidas de control adoptadas han estado inspiradas en la lucha contra el blanqueo de grandes sumas de capital y debemos cuestionarnos si dichos esfuerzos se hacen en el sentido y forma adecuados al problema que nos enfrentamos. Ya hemos comentado anteriormente que la fi- 
nanciación del terrorismo es el reverso del blanqueo de capitales (se utilizan fuentes legales para fines ilegales) y que, cuando la fuente de financiación es ilegal, sólo es necesario su blanqueo cuando el dinero pasa por los canales formales, si las cantidades relativamente pequeñas de dinero no pasan por el sistema bancario formal, la financiación de actividades terroristas no necesita del proceso de blanqueo de capitales, con esconder el origen y destino del dinero es suficiente. En los ejemplos de células españolas desmanteladas, hemos visto como un método muy común para camuflar el origen y destino del dinero es el uso de empresas tapadera ${ }^{45}$. En el caso del dinero negro procedente de la delincuencia y dedicado directamente a acciones delictivas, no es necesaria ninguna pantalla o intermediario que esconda el rastro del dinero, se utilizan correos personales o sistemas informales de transferencia de fondos y se logra el efecto deseado. Además, el hecho que la financiación del terrorismo no busque el ánimo de lucro minimiza las posibilidades de blanqueo de dinero o necesidad de reutilizar el dinero obtenido por fines ilegales para otros usos legales.

Unos de los sectores que se ha visto más vulnerables a posibles abusos por parte de actividades ilegales, dentro de las cuales puede encontrarse el terrorismo es el sector de exportación e importación. De las entrevistas realizadas a oficiales de aduana y del análisis de los datos de las importaciones se desprende que existen lagunas importantes en la forma en que los gobiernos gestionan el sector del comercio. La documentación revisada suele ser incompleta, errónea y, en ciertos casos, ilegal cuando revisamos los formularios que deben rellenar las autoridades aduaneras. Queda mucho por hacer para alcanzar niveles de transparencia en dichas transacciones ${ }^{46}$. Existe, por tanto, importantes oportunidades para las organizaciones terroristas en la utilización de este mercado como forma de procurar financiación y transferir fondos a nivel internacional.

Como consecuencia, es recomendable poner en marcha medidas que mejoren la transparencia y la rendición de cuentas en el comercio de la importación y exportación para poder prevenir y detectar conductas irregulares de financiación que se esconden detrás de las transacciones internacionales. Existe actualmente la tecnología y las herramientas de control para detectar irregularidades, para comparar importaciones y exportaciones, en definitiva, para mejorar el cono-

45 En los casos mencionados, eran empresas gerenciadas por españoles o que realizaban actividades en España que servían para canalizar donaciones y dinero procedente de fuentes ilegales para desviarlo a otros fines ilegales.

46 Ver Passas (2004) para más detalles. 
cimiento sobre las irregularidades y sus causas ${ }^{47}$. La efectiva aplicación de estos métodos servirá de gran ayuda para detectar anomalías y prevenir conductas de riesgo futuras. Una medida de acción muy útil sería la creación de departamentos para la transparencia del comercio en los distintos países, para poder crear estrategias conjuntas y coordenadas de control y de detección precoz de irregularidades. Asimismo, la cooperación privada que tanto se ha buscado en el control del sistema financiero también sería deseable en el sector del comercio. La iniciativa privada sería un colaborador privilegiado para mejorar la prevención y detección de infracciones.

\section{Conclusiones}

Tal como hemos visto a lo largo de esta exposición, la financiación del terrorismo de los grupos asociados a al Qaida tiene su origen, por un lado, en el apoyo social de ciertos sectores de la población que, directa o indirectamente, ofrecen apoyo a estos grupos y, por otro, en las relaciones con distintas actividades delincuenciales que se utilizan como canales de obtención de fondos. Así, actividades legales e ilegales se relacionan en una simbiosis difícil de desentramar y donde el proceso de blanqueo es muchas veces innecesario, ya que el fin de estas organizaciones no es reutilizar dicho dinero para otras actividades legales, sino utilizar el excedente para mantener al mayor número de militantes posible y realizar otros actos de terrorismo en otros países.

La lucha contra la financiación del terrorismo ha venido de la mano de la lucha contra el blanqueo de capitales, desconociendo exactamente cuál es el objetivo y qué forma tenía. El control de la financiación debe priorizar el conocimiento empírico para poder adecuar mejor sus medidas de control. Hasta ahora, esta lucha ha sido desproporcionada, priorizando medidas preventivas y minimizadoras del riesgo. Desde el atentado del 11 de septiembre, se ha detectado una mayor utilización de los sistemas informales de transferencias de fondos para ocultar el rastro del dinero y mayor acceso a actividades delictivas como forma de autofinanciación. Todo control de dichos sistemas informales debe tener en cuenta el interés de la población a la que sirven y los usuarios que utilizan legítimamente estos sistemas.

Medidas de control a corto plazo deben combinarse con medidas a largo plazo que ayuden a una mejor comprensión de las razones por las cuales se milita en estos grupos, qué motivos culturales, sociales y psi-

47 Ver Passas (2006b). 
cológicos generan la cohesión de los grupos, qué factores incrementan su radicalización, etc. Estas son las raíces del problema y su abordaje puede ser la única solución. Solo así conseguiremos disminuir su seguimiento y diseñar medidas de reducción del número de miembros y simpatizantes. Medidas de control que no se sustenten en un conocimiento profundo de estos aspectos no sólo serán ineficaces sino contraproducentes para nuestros intereses. Toda medida de control debe tener en cuenta el impacto que produce en la población y las consecuencias que puede tener para ella, con el objetivo de minimizar el sentimiento de venganza y de rechazo que puede provocar una aplicación indiscriminada de medidas fundadas en una concepción puramente occidental.

Las acciones de control de la financiación se han centrado en la búsqueda de grandes volúmenes de dinero y grandes fuentes de financiación en el ámbito internacional. A través de los distintos ejemplos, hemos visto cómo puede hacerse mucho daño consiguiendo financiación de carácter local y que es a estos niveles donde podemos detectar las actividades de movimiento de fondos y financiación que requieren estos grupos. La colaboración con las entidades privadas, el fomento de la integración de la comunidad musulmana, la profundización de la relación que existe entre redes de delincuencia organizada y terrorismo son pistas para ahondar en el seguimiento de estos movimientos.

La colaboración con el sector privado para detectar transacciones irregulares es un elemento primordial que debe fomentarse y promoverse. Sin embargo, hay un sector todavía poco controlado que es el del comercio que, donde se producen muchas irregularidades y las medidas de control no se aplican estrictamente. De este modo se convierte en un sector vulnerable y con posibilidades de ser utilizado por terroristas para procurarse financiación. Deberíamos prestar más atención a este sector, mejorando los mecanismos de control ya existentes.

\section{Bibliografía citada}

ABUZA, Z. (2004): «Cómo se financia el terrorismo islamista: la experiencia del sudeste asiático». En Reinares, F. y Elorza, A. (coord.). El nuevo terrorismo islamista. Del 11-S al 11-M. Madrid: Temas de Hoy.

ARISTEGUI, G. (2004): El Islamismo contra el Islam. Las claves para entender el terrorismo yihadista. Barcelona: Ediciones B.

BuRKe, J. (2002): Al Qaida: Casting a Shadow of Terror. New York: I.B. Tauris.

CAMPón Dominguez, J. A. (2004): «El Blanqueo de capitales y la financiación del terrorismo como amenazas de la seguridad». Cuadernos de la Guardia Civil, n. ${ }^{\circ}$. 24, pp. 13-24. 
Dandurand, Y., y ChIn, V. (2004): Links between Terrorism and Other Forms of Crime. Report to Foreign Affairs Canada and The United Nations Office on Drugs and Crime. Vancouver.

Escobar Stemman, J. J. (2004): «Cómo luchar contra al Qaida». Política Exterior. Vol. XVIII, n. ${ }^{\circ}$ 99, pp. 17-24.

Financial Action task Force ON MONEy LAUndering (2003): Report on Money Laundering Typologies. Paris. Financial Action Task Force, OECD.

Gunaratna, R. (2002): Inside al-Qaida: Global Network of Terror. New York: Columbia University Press.

HaAs, R. N., Wechsler, W. F., y Wolosky, W. F. (2004): Report of the Independent Task Force on Terrorism Financing. Nueva York: Council on Foreign Relations.

LEE, R. (2002): Terrorist financing: The US and International Response. Washington, DC: Congressional Research Service.

LeVITT, M. (2002): Terrorism Financing: Report of an Independent Task Force Sponsored by the Council of foreign Relations. New York: The Council of Foreign Relations.

Maimbo, S. M. (2003): The Money Exchange Dealers of Kabul. A Study of the Hawala System in Afghanistan. Washington, DC: The World Bank.

National Commission on Terrorist Attacks upon the United States (2004): Monograph on Terrorist Financing. Washington, DC: National Commission on Terrorist Attacks upon the United States.

NAYLOR, R. T. (2002): Wages of Crime: Black Markets, Illegal Finance, and the Underground Economy. Ithaca and London: Cornell University Press.

NaYlor, R. T. (2006): Satanic Purses: Money, Myth, and Misinformation in the War on Terror. Montreal and Kingston: McGill-Queen University Press

PASSAS, N. (2005): «Formalizing the Informal? Problems in the National and International Regulation of Hawala». Regulatory Frameworks for Hawala and Other Remittance Systems, pp. 7-16. Washington, DC: IMF.

- (2007): «Terrorism Financing Mechanisms and Policy Dilemmas». En Trinkunas, G y Giraldo, J. The Political Economy of Terrorism Financing and State Responses: A Comparative Perspective. Stanford: Stanford University Press.

- (2006a): «Fighting Terror with Error: The Counter-productive Regulation of Informal Value Transfers». Crime, Law and Social Change, Vol: 45, n. ${ }^{\circ} 4-5$, pp. 315-336.

- (2006b): "Setting Global CFT Standards: A Critique and Suggestions». Journal of Money Laundering Control, Vol: 9. n. ${ }^{\circ}$ 3, pp. 281-292.

- (2006c): Desmystifying Hawala: A Look into its Social Organization and Mechanics. Journal of Scandinavian Studies in Criminology and Crime Prevention. Vol. 7, pp. 46-62.

- (2004): The Trade in Diamonds: Vulnerabilities for Financial Crime and Terrorist Finance. Vienna, Virginia: FinCEN, US Treasury Department.

- (2003a): «Financial Controls of Terrorism and Informal Value Transfer Methods. En Van de Brunt, H., Siegel, D. y Zaitch, D. (Ed.): Transnational Organized Crime. Current Developments. Dordrecht: Kluwer.

- (2003b): «Hawala and Other Value Transfer Systems: How to Regulate Them?» Risk Management. Vol: 5, n. ${ }^{\circ}$ 2. pp. 49-59. 
- (2003c): Informal Value Transfer Systems, Terrorism and Money Laundering. Report to National Institute of Justice and Financial Crimes Enforcement Network. http: //www.ncjrs.org/pdffiles1/nij/grants/208301.pdf.

- (2002): Informal Value Transfer Systems, Money Laundering and Terrorism. Interim Report to Financial Crimes Enforcement Network. The Hague: The Netherlands.

Passas, N., y Jones, K. (2006): «The Trade in Commodities and Terrorist Financing: Focus on Diamonds». European Journal of Criminal Policy and Research. Vol. 12. (disponible en: http: //dx.doi.org/10.1007/s10610-0069006-3), pp. 1-33.

ScHмID, A., Links between terrorist and organized crime networks: emerging patterns and trends. Disponible en: http: //www.iss.co.za/Seminars/terro19sep03/links.pdf.

WeISs, M.A., CRS Report for Congress. Terrorist Financing: The 9/11 Commission Recommendations. 